\title{
Focal Low and Global High Permeability Predict the Possibility, Risk, and Location of Hemorrhagic Transformation following Intra-Arterial Thrombolysis Therapy in Acute Stroke
}

\author{
(D) Y. Li, (D)Y. Xia, (D) H. Chen, (D) N. Liu, (D)A. Jackson, (D) M. Wintermark, (D) Y. Zhang, (D). Hu, (D). Wu, (D). Zhang, (D). Tu,
}

(D) Z. Su, and (D) G. Zhu

O- $\equiv$

\begin{abstract}
BACKGROUND AND PURPOSE: The contrast volume transfer coefficient $\left(K^{\text {trans }}\right)$, which reflects blood-brain barrier permeability, is influenced by circulation and measurement conditions. We hypothesized that focal low BBB permeability values can predict the spatial distribution of hemorrhagic transformation and global high BBB permeability values can predict the likelihood of hemorrhagic transformation.
\end{abstract}

MATERIALS AND METHODS: We retrospectively enrolled 106 patients with hemispheric stroke who received intra-arterial thrombolytic treatment. $K^{\text {trans }}$ maps were obtained with first-pass perfusion CT data. The $K^{\text {trans }}$ values at the region level, obtained with the Alberta Stroke Program Early CT Score system, were compared to determine the differences between the hemorrhagic transformation and nonhemorrhagic transformation regions. The $K^{\text {trans }}$ values of the whole ischemic region based on baseline perfusion CT were obtained as a variable to hemorrhagic transformation possibility at the global level.

RESULTS: Forty-eight (45.3\%) patients had hemorrhagic transformation, and 21 (19.8\%) had symptomatic intracranial hemorrhage. At the region level, there were 82 ROls with hemorrhagic transformation and parenchymal hemorrhage with a mean $K^{\text {trans }}, 0.5 \pm 0.5 /$ min, which was significantly lower than that in the nonhemorrhagic transformation regions $(P<.01)$. The mean $K^{\text {trans }}$ value of 615 nonhemorrhagic transformation ROls was $0.7 \pm 0.6 / \mathrm{min}$. At the global level, there was a significant difference $(P=.01)$ between the mean $K^{\text {trans }}$ values of patients with symptomatic intracranial hemorrhage $(1.3 \pm 0.9)$ and those without symptomatic intracranial hemorrhage $(0.8 \pm 0.4)$. Only a high $K^{\text {trans }}$ value at the global level could predict the occurrence of symptomatic intracranial hemorrhage $(P<.01 ; \mathrm{OR}=5.04 ; 95 \% \mathrm{Cl}$, 2.01-12.65).

CONCLUSIONS: Global high $K^{\text {trans }}$ values can predict the likelihood of hemorrhagic transformation or symptomatic intracranial hemorrhage at the patient level, whereas focal low $K^{\text {trans }}$ values can predict the spatial distributions of hemorrhagic transformation at the region level.

ABBREVIATIONS: $\mathrm{AIS}=$ acute ischemic stroke; $\mathrm{HI}=$ hemorrhagic infarction; $\mathrm{HT}=$ hemorrhagic transformation; $\mid \mathrm{AT}=$ intra-arterial thrombolysis; $K^{\mathrm{krans}}=$ contrast volume transfer coefficient; $\mathrm{PCT}$ = perfusion $\mathrm{CT} ; \mathrm{PH}=$ parenchymal hemorrhage; $\mathrm{sICH}=$ symptomatic intracranial hemorrhage

$\mathrm{H}$ emorrhagic transformation (HT) is a dreaded complication of reperfusion therapy after acute ischemic stroke (AIS). HT is nearly 5 times more likely following intravenous thrombolysis

Received September 7, 2016; accepted after revision May 6, 2017

From the Department of Neurology (Y.L., H.C., N.L., W.Z.), PLA Army General Hospital, Beijing, China; Department of Critical Care Medicine (Y.X.), Yantai

Yuhuangding Hospital, Shandong, China; Wolfson Molecular Imaging Centre (A.J.), University of Manchester, Manchester, UK; Department of Radiology (Y.L., M.W.), Neuroradiology Section, Stanford University, Stanford, California; Department of Neurology (Y.Z.), Changhai Hospital, Second Military Medical University, Shanghai, China; Department of Neurology (J.H., G.Z.), Southwest Hospital, Third Military Medical University, Chongqing, China; Department of Radiology (B.W.), PLA Army General Hospital, Beijing, China; Outpatient Department (J.T.), PLA 61889 Army, Beijing, China; and GE Healthcare (Z.S.), Beijing, China.

Ying Li and Yonghong Xia contributed equally to this study.

This work was supported by the National Natural Science Foundation of China (grant Nos. 81371286 and 81501024) and the Clinical Innovation Foundation of Southwest Hospital (grant No. SWH2013LC20). compared with controls ${ }^{1}$ and even more common following intra-arterial thrombolysis (IAT). ${ }^{2}$ Symptomatic intracranial hemorrhage (sICH), the most serious complication after reperfusion therapy, is associated with an independent increase in mortality at 3 months after acute ischemia stroke. ${ }^{3}$ It is therefore crucial to be able to predict the risks of $\mathrm{HT}$ and $\mathrm{sICH}$ before making a decision to use reperfusion therapy, especially intra-arterial procedures.

Please address correspondence to Guangming Zhu, MD, PhD, Department of Neurology, Southwest Hospital, Third Military Medical University, Chongqing, 400038, China; e-mail: zhugmdc@aliyun.com; Zihua Su, PhD, Advanced Application Department, Life Science, GE Healthcare, Beijing 100176, China; e-mail: zihua.su@qq.com

-- Indicates open access to non-subscribers at www.ajnr.org

三 Indicates article with supplemental on-line tables.

http://dx.doi.org/10.3174/ajnr.A5287 
A number of clinical risk factors, including age, diabetes mellitus, infarction volume, and anticoagulant or antiplatelet therapy, are associated with increased risk of HT. ${ }^{3,4}$ In addition, focal ischemia and reperfusion injury may lead to blood-brain barrier disruption, which is seen as early as 6 hours after infarction and increases with time, ${ }^{5}$ and HT often appears in these areas. Clinical features alone provide poor predictive power for the risk of HT, and recent studies have assessed biomarkers of perfusion and endothelial permeability derived from dynamic contrast-enhanced imaging, specifically the contrast volume transfer coefficient $\left(K^{\text {trans }}\right) .{ }^{6,7} K^{\text {trans }}$ represents the product of endothelial permeability and endothelial surface and governs the leakage of contrast from the vascular to the extravascular compartment. Several of these studies have suggested that increased blood-brain barrier permeability of an ischemic area predisposes to HT after reperfusion therapy. ${ }^{8-11}$

In our previous study, we proposed that $K^{\text {trans }}$, based on the model of Patlak and Blasberg, ${ }^{12}$ derived from standard first-pass perfusion CT (PCT), can predict the relative risk of HT following intra-arterial reperfusion. However, in contrast to other studies, we found that HT was most likely to develop in the areas within the infarct that had lower permeability values. ${ }^{13}$ We hypothesized that this apparent discrepancy arose from previous studies focusing on the mean values of $K^{\text {trans }}$ derived from within the entire infarct volume as a predictive feature for HT, whereas in our previous study, we studied $K^{\text {trans }}$ values measured specifically in areas of the brain that subsequently develop HT. Taken in combination, the studies suggest that HT is more common in infarcts characterized by high mean $K^{\text {trans }}$ but is most likely to occur within the infarcted area in those who develop hemorrhage after IAT therapy, characterized by low values of $K^{\text {trans }}$.

In this study, we tested the hypotheses that in patients with AIS treated with IAT with tPA, high average values of $K^{\text {trans }}$ within the infarct predict the risk of HT or sICH after reperfusion, and focal areas with low $K^{\text {trans }}$ predict the geographic location of HT.

\section{MATERIALS AND METHODS \\ Patients}

The clinical and imaging data presented in this study were extracted from a repository created from data collected as part of standard clinical stroke care at 3 participating institutions: the PLA Army General Hospital, Beijing; Southwest Hospital, Chongqing; and Changhai Hospital, Shanghai, China. The respective institutional review boards approved collection and analysis of data from the repository.

We retrospectively enrolled consecutive patients who were admitted to these institutions with signs and symptoms suggesting hemispheric stroke, from January 2011 to January 2014, who met the following inclusion criteria: National Institutes of Health Stroke Scale scores on admission of 4-22; completion of a stroke CT imaging work-up including noncontrast CT, CT angiography, and PCT on admission; no high density that suggested intracranial hemorrhage on baseline noncontrast CT; treatment with IAT, with $<12$ hours from symptom onset to IAT completion; coagulation function normal before IAT; no anticoagulation or antiplatelet agent given within 24 hours after IAT; and MR imaging

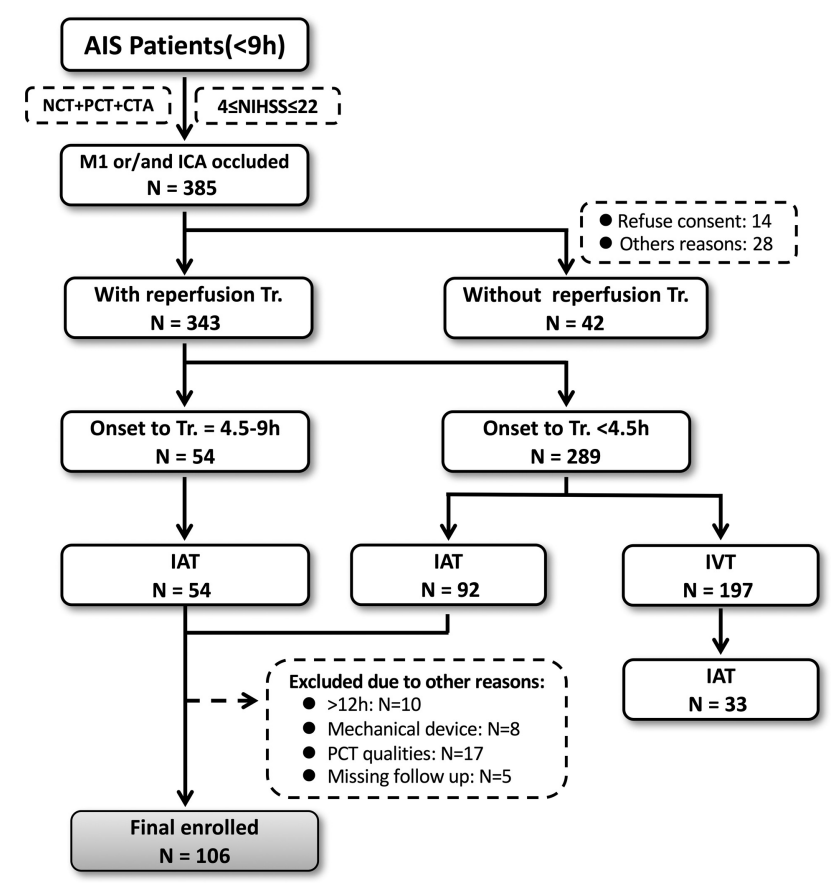

FIG 1. Flow chart outlining patient selection. Tr. indicates treatment; IVT, intravenous thrombolysis; NCT, noncontrast CT.

performed on the third day after therapy. A flow chart outlining the rules for patient selection is shown in Fig 1.

We recorded the following demographic and clinical variables: age, sex, medical history, vascular risk factors, time from symptom onset to treatment, the National Institutes of Health Stroke Scale score on admission, and the modified Rankin Scale score at 90 days. The 90-day outcomes were assessed in an outpatient clinic or over the telephone. An mRS of $\leq 2$ was considered a good clinical outcome. Death was coded as $\mathrm{mRS}=6$.

Stroke mechanisms were subtyped by using the Trial of Org 10172 in Acute Stroke Treatment classification ${ }^{14}$ and were diagnosed by the consensus of 2 stroke neurologists (J.H. and Y.Z.). The sICH was recorded as clinical neurologic worsening or death after therapy caused by parenchymal hemorrhage (PH) or hemorrhagic infarction (HI). ${ }^{15}$

\section{Imaging Protocol}

PCT Image Acquisition. PCT studies were performed on similarly specified 64-section CT scanners (Discovery CT750 HD; GE Healthcare, Milwaukee, Wisconsin). Each PCT study involved successive gantry rotations performed in cine mode during intravenous administration of 2 boluses of $40 \mathrm{~mL}$ of iohexol (Omnipaque 350; GE Healthcare, Piscataway, New Jersey) at an injection rate of $4 \mathrm{~mL} / \mathrm{s}$. First-pass PCT acquisitions ranged from 50 to 70 seconds, with a sampling interval of either 1 or 2 seconds. Total PCT coverage was $40 \mathrm{~mm}$. Acquisition parameters were $80 \mathrm{kVp}$ and $100 \mathrm{mAs}$.

CTA Imaging Protocol. CTA studies of the cervical and intracranial arteries were performed at $120 \mathrm{kVp} / 200-300 \mathrm{mAs}$ with the following acquisition parameters: helical mode; 0.5- to 0.8-second gantry rotation; pitch, 1-.375:1; section thickness, 0.625-1.25 $\mathrm{mm}$; and reconstruction interval, $0.5-1 \mathrm{~mm}$. A caudocranial 


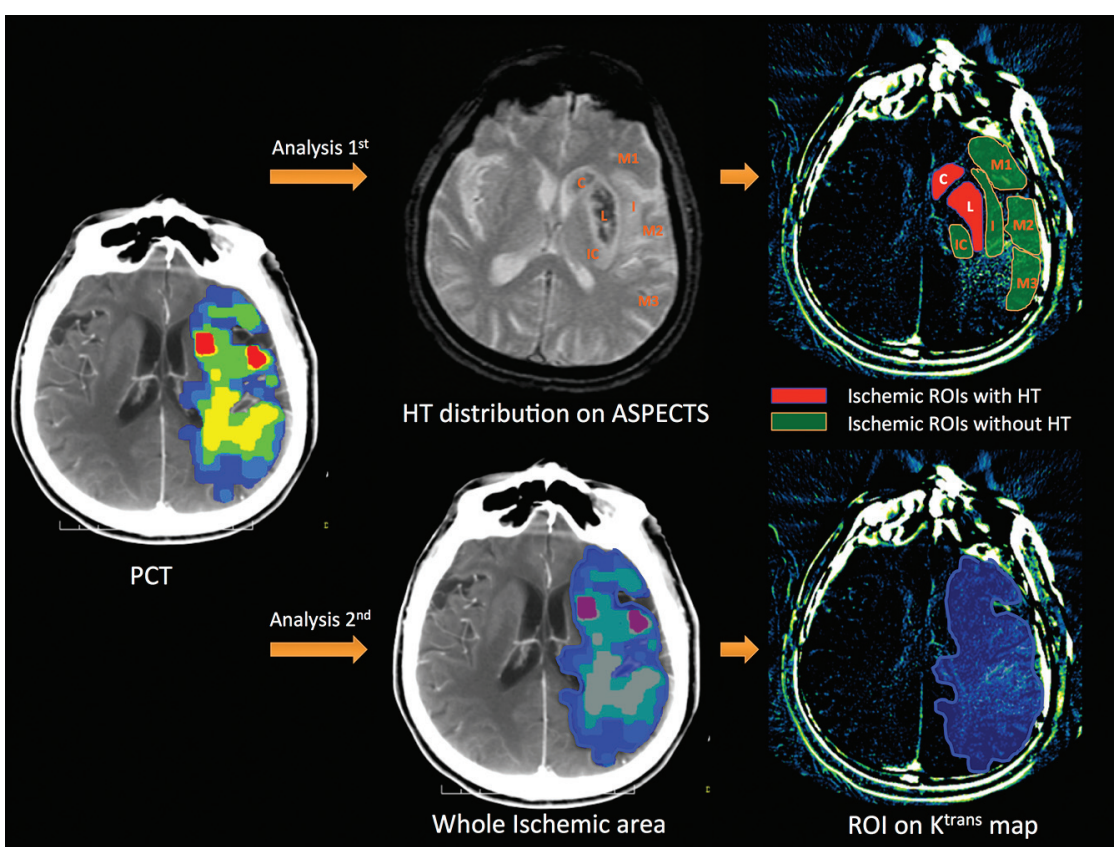

FIG 2. Protocol used to define ROIs (HT area and non-HT area) on $K^{\text {trans }}$ maps. First analysis: the ASPECTS system was used to manually draw the distribution of the ischemic area on $K^{\text {trans }}$ maps and determine the permeability values of each ROI. Second analysis: the entire ischemic area from PCT imaging is overlapped on the $K^{\text {trans }}$ maps to determine the ROI, and the permeability value is restricted to this area.

scanning direction was selected covering the midchest to the vertex of the skull. CTA scans were obtained with $50 \mathrm{~mL}$ of iohexol (Omnipaque 350) administered via power injector at $5 \mathrm{~mL} / \mathrm{s}$.

DSA Imaging Protocol. DSA was performed with a dedicated biplane cerebral angiographic system (Axiom Artis dBA Twin; Siemens, Erlangen, Germany). Images during injection of each internal and external carotid artery and at least 1 vertebral artery were acquired and stored. All patients underwent IAT. Reperfusion status was assessed on DSA after IAT, and successful recanalization was defined as TICI $2 \mathrm{~b}$ and 3 .

MR Imaging Protocol. MR imaging was performed on 1.5T MR imaging scanners (Signa; GE Healthcare). Gradient recalledecho sequence parameters were as follows: TR, $600 \mathrm{~ms}$; TE, 20 $\mathrm{ms}$; flip angle, $20^{\circ}$; matrix size, $256 \times 160$; section thickness, 6 $\mathrm{mm}$; intersection gap, $1 \mathrm{~mm}$; 20 axial sections; and FOV, $250 \times$ $180 \mathrm{~mm}$.

\section{Image Processing}

A neurologist (H.C.) with 13 years of experience reviewed all noncontrast CT, PCT, and CTA data. She measured the Alberta Stroke Program Early CT Score ${ }^{16}$ and the hyperdense middle cerebral artery sign ${ }^{17}$ on noncontrast $\mathrm{CT}$ and the infarct core and penumbra volumes on PCT. A neurologist (N.L.) with 14 years of experience reviewed the follow-up MR images to measure the final infarct volumes. Both reviewers were blinded to the initial clinical interpretation of these studies and were provided with only the side of the signs and symptoms.

Conventional perfusion parameters were calculated from PCT data with the commercially available software MIStar (Apollo Medical Imaging Technology, Melbourne, Australia).
The software applies singular value decomposition with delay- and dispersion-correction to calculate parametric perfusion maps and to automatically measure the volumes of infarct and penumbra. ${ }^{18}$ Cerebral tissue with delay time $>3$ seconds, which includes penumbra and infarct core, was integrated as ischemic. ${ }^{19}$ Parametric maps of $K^{\text {trans }}$ were calculated from PCT data with prototype software (Omni-Kinetics; GE Healthcare, Beijing, China), ${ }^{20}$ which is based on the model of Patlak et al. ${ }^{21}$ $K^{\text {trans }}$ maps were loaded into ImageJ 1.47 for Mac OS (National Institutes of Health, Bethesda, Maryland). A neurologist (Y.L.) defined ROIs with a manual drawing based on PCT, deriving mean $K^{\text {trans }}$ values for each ROI.

HT was defined on the basis of the gradient-echo $\mathrm{T}^{*}$-weighted ${ }^{22}$ images obtained on day 3, using the European Cooperative Acute Stroke Study ${ }^{23}$ classification of hemorrhagic infarct: 1) no HT; 2) HI; and 3) PH.

Here we built 2 levels of permeability values: a region level and global level. In the region level, $K^{\text {trans }}$ values of all patients were assessed according to the ASPECTS system. The 10 ASPECTS regions included the following: caudate, lenticula, internal capsule, insular ribbon, and M1-M6. On the basis of the PCT data at admission and the follow-up gradient-echo $\mathrm{T} 2{ }^{*}$-weighted images, the ASPECTS regions in each patient were divided into 4 categories: 1) ischemic tissue without HT (simplified as non-HT);2) ischemic tissue with $\mathrm{HI}$ (simplified as $\mathrm{HI}$ ); 3 ) ischemic tissue with $\mathrm{PH}$ (simplified as $\mathrm{PH}$ ); and 4) normal tissue without ischemia (simplified as normal). The $K^{\text {trans }}$ values in the 3 categories, including the ischemic areas with non-HT, $\mathrm{HI}$, or $\mathrm{PH}$, were measured. Of note, here we did not include the normal tissue without ischemia. The $K^{\text {trans }}$ values within non-HT areas were calculated as controls. The protocol used to define the ROIs on the basis of the ASPECTS system is illustrated in Fig 2.

In the global level, the ROIs were drawn on the basis of the whole ischemic regions observed on the output imaging of PCT, as described above; these ROIs were then imported into the $K^{\text {trans }}$ maps to measure the permeability values of the entire ischemic region. The protocol used to define the ROIs for the entire ischemic region is illustrated in Fig 2. The mean permeability values in ischemic tissue for each patient were calculated and recorded because there were multiple sections on PCT imaging.

\section{Outcome Measurements}

There were 3 outcomes in this study: 1) the presence or absence of HT based on follow-up imaging data, 2) the occurrence (or absence) of sICH, and 3 ) favorable ( $\mathrm{mRS}>2$ ) versus unfavorable $(\mathrm{mRS} \leq 2)$ clinical outcome. 


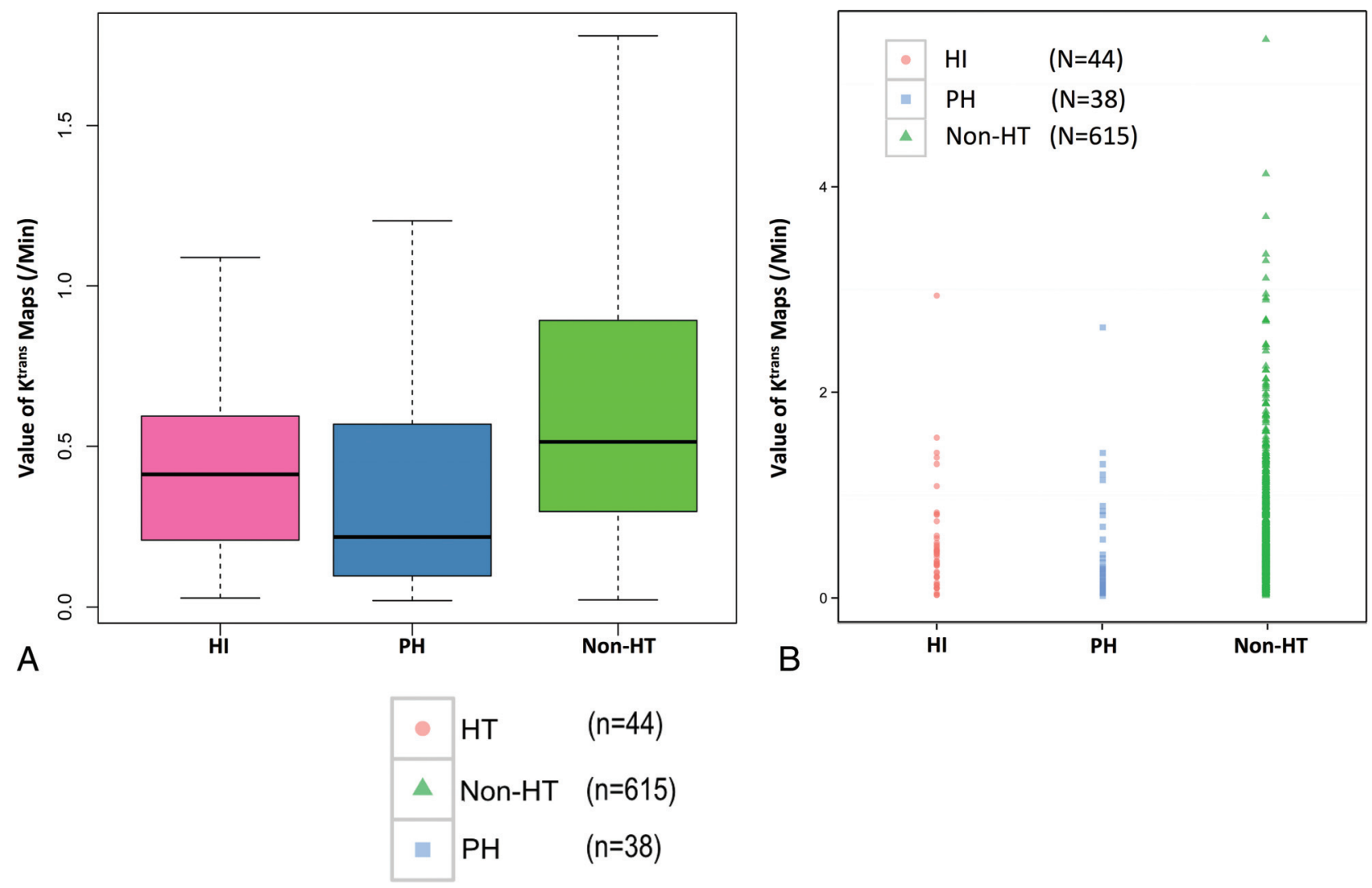

FIG 3. Boxplots and value distribution of $K^{\text {trans }}$ values in the ASPECTS regions. A, Boxplots of $K^{\text {trans }}$ values in the HI, PH, and non-HT ASPECTS regions. In each box, the median, $95 \% \mathrm{Cl}$, and first and third quartile values are illustrated. $B$, The graph illustrates the distribution of the $K^{\text {trans }}$ values, grouped into the $\mathrm{HI}, \mathrm{PH}$, and non-HT regions.

\section{Statistical Analyses}

All data were presented as mean, median with interquartile range, or number with percentages. The baseline characteristics were compared on the basis of different outcomes such as HT, sICH, and good clinical outcome. Age and systolic and diastolic blood pressure on admission were analyzed with Student $t$ tests. The time from symptom onset to CT, NIHSS score on admission, ASPECTS, collateral flow score, percentage of carotid artery stenosis, PCT infarct core, PCT ischemic penumbra, permeability value of the HT regions, permeability value of the non-HT regions, and $\mathrm{mRS}$ at 90 days $(\leq 2)$ were analyzed with Wilcoxon rank sum tests. The Pearson $\chi^{2}$ test was used to analyze the relationship between stroke subtypes and the sites of occlusion. The effect of sex, hypertension, diabetes, hyperlipidemia, atrial fibrillation, current smoking, and the use of statins on admission was compared using the Pearson $\chi^{2}$ test with a Yates continuity correction. Coronary heart disease and recanalization were analyzed with the Fisher exact test.

At the region level, mean $K^{\text {trans }}$ values of ROIs with $\mathrm{HI}, \mathrm{PH}$, and non-HT based on the ASPECTS system were compared using the Kruskal-Wallis rank sum test. Mean $K^{\text {trans }}$ values of the HT (HI plus $\mathrm{PH}$ ) and non-HT ischemic regions were compared using the Wilcoxon rank sum test and a receiver operating characteristic curve.

At the global level, mean $K^{\text {trans }}$ values of whole ischemic regions were entered into 3 multiple logistic regression analyses to determine whether they were independent predictors of HT,
sICH, and good clinical outcome. We entered the baseline predictors into multivariate models in cases with $P<.05$ in the univariate analysis. The results were reported as odds ratios with $95 \%$ confidence intervals. All statistical analyses were performed with SAS, Version 9.3 (SAS Institute, Cary, North Carolina). A $P$ value $<.05$ was considered statistically significant.

\section{RESULTS}

Our study population consisted of 106 patients, including 47 $(44.3 \%)$ women; the mean age was $65.3 \pm 13.9$ years. Eighty-six (81.1\%) patients had successful recanalization. Of the 48 (45.3\%) patients with HT, 21 (19.8\%) had sICH. Moreover, 54 (50.9\%) patients had a good clinical outcome $(\mathrm{mRS}<2)$. The details of clinical and imaging characteristics and outcome are described in On-line Tables 1 and 2.

The first analysis considered a total of 697 ischemic ROIs determined on the basis of the ASPECTS system. Of these ROIs, 44 had HI on follow-up and a mean $K^{\text {trans }}$ value of $0.5 \pm 0.5 / \mathrm{min}$, whereas 38 showed $\mathrm{PH}$ on follow-up and a mean $K^{\text {trans }}$ value of $0.4 \pm 0.5 / \mathrm{min}$. The mean $K^{\text {trans }}$ value of 615 non-HT ROIs was $0.7 \pm 0.6 / \mathrm{min}($ Fig 3$)$. The mean $K^{\text {trans }}$ value of the ROIs in the patients with $\mathrm{HT}$ and $\mathrm{PH}(0.5 \pm 0.5 / \mathrm{min})$ was significantly lower than that in the non-HT regions $(0.7 \pm 0.6 / \mathrm{min}, P<.01$, Fig $4 A)$. The mean $K^{\text {trans }}$ value of the normal tissue ROIs was $0.2 \pm 0.2 /$ min. These HT distributions were most commonly located in the lenticular nucleus (25/82) based on the ASPECTS system, with a mean $K^{\text {trans }}$ value of $0.2 \pm 0.2$. Other locations included the insu- 

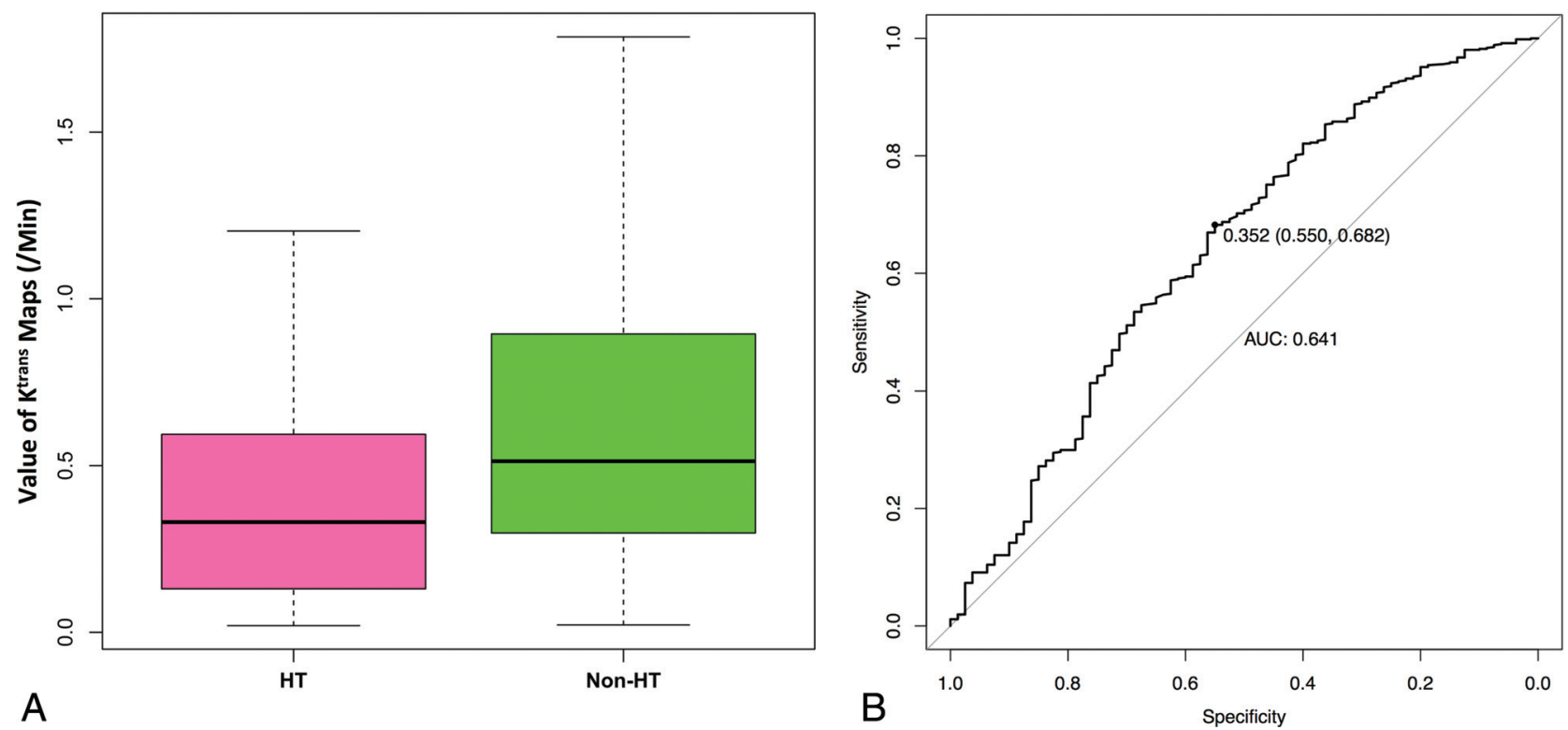

FIG 4. Boxplots of $K^{\text {trans }}$ values of $\mathrm{HT}$ and non-HT regions and the receiver operating characteristic curve. $A$, Boxplots of $K^{\text {trans }}$ values in $\mathrm{HT}$ and non-HT regions. In each box, the median, $95 \% \mathrm{Cl}$, and first and third quartile values are illustrated. $B$, The optimal cutoff value of the $K^{\text {trans }}$ value for the prediction of $\mathrm{HT}$ is identified with receiver operating characteristic curve analysis.

Predictor variables determined to be uniquely associated with different outcomes

\begin{tabular}{|c|c|c|c|c|c|c|c|}
\hline Outcomes & Variable & Factors & Standard Error & Wald $\chi^{2}$ & $P$ Value & OR & OR $95 \% \mathrm{Cl}$ \\
\hline \multirow[t]{3}{*}{ HT vs non-HT } & Intercept & 2.8 & 1.2 & 5.9 & .02 & & \\
\hline & ASPECTS & -0.5 & 0.1 & 11.2 & .00 & 0.6 & $0.5-0.8$ \\
\hline & $K^{\text {trans }}$ value at patient level & 1.0 & 0.4 & 5.4 & .02 & 2.7 & $1.2-6.5$ \\
\hline \multirow[t]{2}{*}{ sICH vs non-sICH } & Intercept & -3.0 & 0.6 & 28.8 & .00 & & \\
\hline & $K^{\text {trans }}$ value at patient level & 1.6 & 0.5 & 11.9 & .00 & 5.0 & $2.0-12.7$ \\
\hline \multirow[t]{4}{*}{$m R S \leq 2$ vs $m R S>2$} & Intercept & -4.6 & 1.6 & 8.9 & .00 & & \\
\hline & Age & 0.1 & 0.0 & 13.5 & .00 & 1.1 & $1.0-1.1$ \\
\hline & NIHSS score on admission & 0.1 & 0.1 & 6.2 & .01 & 1.1 & $1.0-1.3$ \\
\hline & Recanalization & -2.6 & 0.8 & 11.2 & .00 & 0.1 & $0.0-0.3$ \\
\hline
\end{tabular}

lar lobe $(16 / 82)$ with a mean $K^{\text {trans }}$ value of $0.6 \pm 0.5$ and the caudate nuclei $(15 / 82)$ with a mean $K^{\text {trans }}$ value of $0.2 \pm 0.1$, but there were very few in the capsula interna $(2 / 82)$ with a mean $K^{\text {trans }}$ value of $0.3 \pm 0.2$. Receiver operating characteristic curve analysis indicated an optimal cutoff value for $K^{\text {trans }}$ of $0.35 / \mathrm{min}$, which produced a mean area under the curve of $0.64 \pm 0.06$. The sensitivity and specificity at this threshold were $55.0 \%$ and $68.2 \%$, respectively (Fig $4 B$ ).

The first logistic regression model for the prediction of HT indicated that the ASPECTS was the most powerful independent significant predictor $(P<.01, \mathrm{OR}=0.61 ; 95 \% \mathrm{CI}, 0.46-0.82)$. The $K^{\text {trans }}$ value was also a significant independent predictive factor $(P=.02, \mathrm{OR}=2.75 ; 95 \% \mathrm{CI}, 1.17-6.46)$.

The second logistic regression model for the prediction of sICH showed that only the $K^{\text {trans }}$ value was a significant independent predictive factor $(P<.01, \mathrm{OR}=5.04 ; 95 \% \mathrm{CI}, 2.01-12.65)$. Both models suggested that an overall increase in the $K^{\text {trans }}$ values in the ischemic regions predicted a higher probability of HT or sICH.

The third logistic regression model for the prediction of clinical outcome did not show that the $K^{\text {trans }}$ value was an independent predictor. Instead, age was the most significant predictive factor $(P<.01, \mathrm{OR}=1.08 ; 95 \% \mathrm{CI}, 1.03-1.12)$ for clinical outcome. The NIHSS scores on admission and recanalization were also found to be predictive factors $(P=.01, \mathrm{OR}=1.14 ; 95 \% \mathrm{CI}$, 1.03-1.26; and $P<.01, \mathrm{OR}=0.08$; 95\% CI, 0.03-0.34, respectively). The details are shown in the Table.

\section{DISCUSSION}

AIS is the leading cause of mortality in China and is the second most common cause of mortality worldwide. ${ }^{24,25}$ Recent studies suggest that endovascular reperfusion therapy during the 6-hour time window, ${ }^{26,27}$ or even during the 12-hour time window, can be effective for patients with proximal intracranial artery occlusion. However, HT remains a dangerous complication after reperfusion therapy. Furthermore, sICH- one of the most serious types of HT after AIS-increases the risk of stroke morbidity and mortality. ${ }^{28}$ The prediction of HT or sICH before making a decision about reperfusion therapy could have important implications.

In the present study, 106 patients with AIS received IAT therapy. Moreover, 48 patients developed HT and 21 patients developed sICH. The $K^{\text {trans }}$ values in the entire ischemic region were higher in patients with HT or sICH on follow-up compared with those without $\mathrm{HT} / \mathrm{sICH}$, which is consistent with previous studies. ${ }^{10}$

Many factors are associated with HT after AIS, including age, pretreatment NIHSS, ASPECTS, sex, blood pressure, and diabetes 
mellitus. Recent studies focused on multimodal imaging, including perfusion and permeability imaging, to predict HT. ${ }^{6,7}$ In fact, ischemia and reperfusion injury may lead to BBB disruption, and HT often develops in the area with BBB disruption. BBB disruption can develop as early as 6 hours after cerebral infarction, and the severity increases with time. ${ }^{5}$ Moreover, first-pass dynamic PCT can be used to assess BBB permeability. ${ }^{10}$ Another study using PCT imaging indicated that pretreatment BBB permeability measurement had a sensitivity of $100 \%$ and specificity of $79 \%$ in the prediction of sICH and malignant edema. ${ }^{11}$ In fact, abnormal pretreatment permeability may be associated with an increased risk of $\mathrm{PH}$ following recanalization therapy, not only in patients with acute anterior circulation ischemic stroke but also in patients with acute posterior circulation ischemic stroke. ${ }^{29}$

However, our previous study, which included 41 patients with AIS with HT, showed that a low $K^{\text {trans }}$ value predicted the site where HT would occur with perfect sensitivity and specificity. ${ }^{13}$ Our interpretation of this result is that HT is more likely to develop in areas with the most severe hypoperfusion and ischemic injury, and that the $K^{\text {trans }}$ values are low despite a significant elevation of $\mathrm{BBB}$ permeability. The main reason of the mismatch between the $K^{\text {trans }}$ value and BBB permeability is that the blood flow and delivery of contrast agent are severely limited in the infarct core; therefore, the permeability of these areas could not be estimated correctly from a $K^{\text {trans }}$ map. This finding is consistent with that of studies that suggest that very low cerebral blood volume, ${ }^{30}$ admission PCT-based hypoperfused tissue volume, ${ }^{31}$ time-to-maximum of $>8$ seconds, and lesion volumes are all independent predictors of $\mathrm{PH}$ in patients with acute stroke. ${ }^{32}$ All these studies suggest that the low perfusion areas or severely infarcted tissue is more likely to develop HT after reperfusion therapy. In the current study, we compared the difference in the $K^{\text {trans }}$ values between non-HT and HT areas on the basis of the 10 regions derived from the ASPECTS system. In the present study as well, the regions with low $K^{\text {trans }}$ values indicated the location of HT, though the sensitivity and specificity (55.0\% and 68.2\%) were not as suitable as in our previous study. Nevertheless, the threshold of $K^{\text {trans }}$ for predicting HT in the present study $(0.35 /$ $\mathrm{min})$ was similar to that in the previous study $(0.33 / \mathrm{min})$.

When assessing the overall BBB permeability values, including those of the entire ischemic area (rather than specific regions where HT developed), the patients with HT had significantly higher mean $K^{\text {trans }}$ values compared with those without HT. The difference was even more significant when comparing patients with sICH with those without sICH. Although the $K^{\text {trans }}$ value did not predict the clinical outcome, it was still a significant predictor for $\mathrm{HT}$ or sICH. Our interpretation of this finding is that the severity of BBB disruption reflects the severity of ischemia and thus predicts the likelihood of HT. Age, NIHSS score on admission, and recanalization are significant predictors of 90 -day $\mathrm{mRS}$ values; this finding is consistent with those in previous studies. In the present study, we have also observed that the ASPECTS is a predictor of HT. This finding is consistent with that in another study that demonstrated that a decreased ASPECTS/diffusion-weighted imaging score increased the likelihood of HT after thrombolysis. ${ }^{3}$

The present study has several limitations. First, this is a retrospective analysis with a study population of only 106 cases. Sec- ond, we used only 1 permeability parameter, $K^{\text {trans }}$, to characterize the BBB permeability. Other permeability parameters, such as permeability surface-area product and vascular permeability, could also have been assessed. We used first-pass data from PCT, which is not the standard method for the assessment of BBB permeability. These data are more sensitive to noise and more easily overestimated because of the delayed flow in ischemic regions. ${ }^{31}$ All the above could be reasons that the receiver operating characteristic curve in this study is not great at differentiating HT from non-HT. Further study may need more carefully selected candidates with other more meticulous methods, such as delayed-acquisition perfusion CT or dynamic contrast-enhanced MR imaging. Last, the results from the data base with intra-arterial thrombolysis cannot be generalized to other reperfusion therapies such as IV thrombolysis and mechanical recanalization. However, IAT is still an effective method in the clinical setting to assess patients in the hyperacute phase of AIS within limited reperfusion time windows.

\section{CONCLUSIONS}

This study demonstrates that global high $K^{\text {trans }}$ values may be one of the predictors of the likelihood of HT or sICH and that focal low $K^{\text {trans }}$ values predict the spatial distribution of HT after intraarterial reperfusion treatment. Further studies are needed to examine the role of permeability imaging in the decision-making process in patients with AIS.

\section{ACKNOWLEDGMENTS}

We thank Statistical Elite (http://www.tjstat.com/) for statistical analysis and Editage (https://www.editage.cn/) for language editing.

Disclosures: Max Wintermark—UNRELATED: board membership: GE NFL Advisory Board.

\section{REFERENCES}

1. Hacke W, Donnan G, Fieschi C, et al; ATLANTIS Trials Investigators, ECASS Trials Investigators, NINDS rt-PA Study Group Investigators. Association of outcome with early stroke treatment: pooled analysis of ATLANTIS, ECASS, and NINDS rt-PA stroke trials. Lancet 2004;363:768-74 CrossRef Medline

2. O’Rourke K, Berge E, Walsh CD, et al. Percutaneous vascular interventions for acute ischaemic stroke. Cochrane Database Syst Rev 2010:CD007574 CrossRef Medline

3. Hong KS, Kang DW, Koo JS, et al. Impact of neurological and medical complications on 3-month outcomes in acute ischaemic stroke. Eur J Neurol 2008;15:1324-31 CrossRef Medline

4. Zhang PL, Wang YX, Chen Y, et al. Analysis on the correlation factors for hemorrhagic transformation after intravenous thrombolytic therapy. Eur Rev Med Pharmacol Sci 2015;19:1001-08 Medline

5. Moriya Y, Takahashi W, Kijima C, et al. Predictors for hemorrhagic transformation with intravenous tissue plasminogen activator in acute ischemic stroke. Tokai J Exp Clin Med 2013;38:24-27 Medline

6. Jiang Q, Ewing JR, Chopp M. MRI of blood-brain barrier permeability in cerebral ischemia. Transl Stroke Res 2012;3:56-64 CrossRef Medline

7. Jain AR, Jain M, Kanthala AR, et al. Association of CT perfusion parameters with hemorrhagic transformation in acute ischemic stroke. AJNR Am J Neuroradiol 2013;34:1895-900 CrossRef Medline

8. Ozkul-Wermester O, Guegan-Massardier E, Triquenot A, et al. Increased blood-brain barrier permeability on perfusion computed 
tomography predicts hemorrhagic transformation in acute ischemic stroke. Eur Neurol 2014;72:45-53 CrossRef Medline

9. Campbell BC, Christensen S, Parsons MW, et al; EPITHET and DEFUSE Investigators. Advanced imaging improves prediction of hemorrhage after stroke thrombolysis. Ann Neurol 2013;73:510-19 CrossRef Medline

10. Lin K, Kazmi KS, Law M, et al. Measuring elevated microvascular permeability and predicting hemorrhagic transformation in acute ischemic stroke using first-pass dynamic perfusion CT imaging. AJNR Am J Neuroradiol 2007;28:1292-98 CrossRef Medline

11. Hom J, Dankbaar JW, Soares BP, et al. Blood-brain barrier permeability assessed by perfusion CT predicts symptomatic hemorrhagic transformation and malignant edema in acute ischemic stroke. AJNR Am J Neuroradiol 2011;32:41-48 CrossRef Medline

12. Patlak CS, Blasberg RG. Graphical evaluation of blood-to-brain transfer constants from multiple-time uptake data: generalizations. J Cereb Blood Flow Metab 1985;5:584-90 CrossRef Medline

13. Chen H, Liu N, Li Y, et al. Mismatch of low perfusion and high permeability predicts hemorrhagic transformation region in acute ischemic stroke patients treated with intra-arterial thrombolysis. Sci Rep 2016;6:27950 CrossRef Medline

14. Adams HP Jr, Bendixen BH, Kappelle LJ, et al; TOAST Investigators. Classification of subtype of acute ischemic stroke: definitions for use in a multicenter clinical trial. Stroke 1993;24:35-41 Medline

15. Berger C, Fiorelli M, Steiner T, et al. Hemorrhagic transformation of ischemic brain tissue: asymptomatic or symptomatic? Stroke 2001; 32:1330-35 CrossRef Medline

16. Barber PA, Demchuk AM, Zhang J, et al. Validity and reliability of a quantitative computed tomography score in predicting outcome of hyperacute stroke before thrombolytic therapy: ASPECTS Study Group-Alberta Stroke Programme Early CT Score. Lancet 2000; 355:1670-74 CrossRef Medline

17. Man S, Hussain MS, Wisco D, et al. The location of pretreatment hyperdense middle cerebral artery sign predicts the outcome of intraarterial thrombectomy for acute stroke. J Neuroimaging 2015;25: 263-68 CrossRef Medline

18. Yang Q, inventor; Apollo Medical Imaging Technology Pty Ltd, assignee. Method and system for mapping tissue status of acute stroke. US patent 8942451. January 27, 2015

19. Bivard A, Levi C, Krishnamurthy V, et al. Perfusion computed tomography to assist decision making for stroke thrombolysis. Brain 2015;138(pt 7):1919-31 CrossRef Medline

20. Sourbron SP, Buckley DL. Classic models for dynamic contrast-enhanced MRI. NMR Biomed 2013;26:1004-27 CrossRef Medline

21. Patlak CS, Blasberg RG, Fenstermacher JD. Graphical evaluation of blood-to-brain transfer constants from multiple-time uptake data. J Cereb Blood Flow Metab 1983;3:1-7 CrossRef Medline

22. Arnould MC, Grandin CB, Peeters A, et al. Comparison of CT and three MR sequences for detecting and categorizing early ( 48 hours) hemorrhagic transformation in hyperacute ischemic stroke. AJNR Am J Neuroradiol 2004;25:939-44 Medline

23. Hacke W, Kaste M, Fieschi C, et al. Randomised double-blind placebo-controlled trial of thrombolytic therapy with intravenous alteplase in acute ischaemic stroke (ECASS II): Second EuropeanAustralasian Acute Stroke Study Investigators. Lancet 1998;352: 1245-51 CrossRef Medline

24. Goldstein LB, Bushnell CD, Adams RJ, et al; American Heart Association Stroke Council, Council on Cardiovascular Nursing, Council on Epidemiology and Prevention, Council for High Blood Pressure Research, Council on Peripheral Vascular Disease, and Interdisciplinary Council on Quality of Care and Outcomes Research. Guidelines for the primary prevention of stroke: a guideline for healthcare professionals from the American Heart Association/American Stroke Association. Stroke 2011;42:517-84 CrossRef Medline

25. Wang YJ, Zhang SM, Zhang L, et al. Chinese guidelines for the secondary prevention of ischemic stroke and transient ischemic attack 2010. CNS Neurosci Ther 2012;18:93-101 CrossRef Medline

26. Gobert F, Cho TH, Desilles JP, et al. Magnetic resonance imagingbased intravenous thrombolysis 6 hours after onset of minor cerebellar stroke. Arch Neurol 2011;68:678 CrossRef Medline

27. Newman DH, Shreves AE. Thrombolysis in acute ischaemic stroke. Lancet 2012;380:1053-54; author reply 1054-55 CrossRef Medline

28. Whiteley WN, Thompson D, Murray G, et al; IST-3 Collaborative Group. Targeting recombinant tissue-type plasminogen activator in acute ischemic stroke based on risk of intracranial hemorrhage or poor functional outcome: an analysis of the third international stroke trial. Stroke 2014;45:1000 -06 CrossRef Medline

29. Lee M, Saver JL, Alger JR, et al. Blood-brain barrier permeability derangements in posterior circulation ischemic stroke: frequency and relation to hemorrhagic transformation. J Neurol Sci 2012;313: 142-46 CrossRef Medline

30. Hermitte L, Cho TH, Ozenne B, et al. Very low cerebral blood volume predicts parenchymal hematoma in acute ischemic stroke. Stroke 2013;44:2318-20 CrossRef Medline

31. Schneider T, Hom J, Bredno J, et al. Delay correction for the assessment of blood-brain barrier permeability using first-pass dynamic perfusion CT. AJNR Am J Neuroradiol 2011;32:E134-38 CrossRef Medline

32. Yassi N, Parsons MW, Christensen S, et al. Prediction of poststroke hemorrhagic transformation using computed tomography perfusion. Stroke 2013;44:3039-43 CrossRef Medline 